\title{
My approach to the diagnosis of congenital coronary anomalies by transthoracic echocardiography
}

Como eu Faço o Diagnóstico das Anomalias Congênitas das Artérias Coronárias pelo Ecocardiograma Transtorácico?

Labatt Heart Center; ${ }^{1}$ The Hospital for Sick Children; ${ }^{2}$ University of Toronto, ${ }^{3}$ Toronto, Canada.

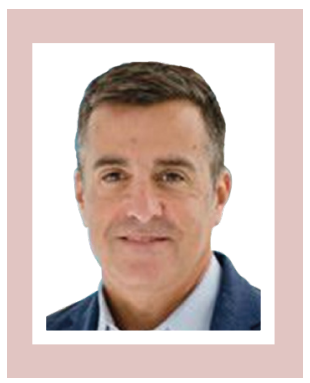

Vitor Coimbra Guerra ${ }^{1,2,3}$

"Your vision will become clear, only when you can look into your heart. Who looks outside: dreams, who looks inside: awakes" (Carl Jung)

\section{Why do we need to assess the anatomy of the coronary arteries?}

Until a few decades ago, coronary artery anatomy assessment by echocardiogram $(\mathrm{ECHO})$ was considered a difficult secondary task even if analyzed by other cardiovascular imaging modalities, such as angiography by cardiac catheterization. Important changes in this approach in recent years can be explained by three important aspects:1) The origin and initial course of coronary artery anomalies can be a substrate for ischemia and even sudden death in young adults; ${ }^{1,2} 2$ ) Transthoracic echocardiography (TTE) is a noninvasive radiation-free low-cost imaging test in the frontline of cardiac assessments;3) The anatomical and functional assessment of coronary arteries is part of a transthoracic test used to diagnose congenital heart diseases. ${ }^{3,4}$

\section{Which congenital coronary artery anomalies are relevant and should be identified?}

The classification of congenital coronary artery anomalies can be extensively sub-categorized by their anatomical and

\section{Keywords}

coronary artery, congenital, echocardiogram.

Mailing Address: Vitor Coimbra Guerra •

555 University Ave, Toronto ON M5G 1X8

E-mail: vitor.guerra@sickkids.ca

Manuscript received 10/14/2021; revised 10/16/2021; accepted 10/21/2021

DOI: 10.47593/2675-312X/20213403ecom16 hemodynamic presentation. Historically, they were simply classified as major versus minor anomalies. Currently, a more comprehensive, broad, and detailed classification covers all anatomical variation possibilities. ${ }^{5}$

The first step is to know the normal pattern (Table 1) from a quantitative and qualitative point of view. Once the normal pattern is excluded, all findings should be considered for the definitive diagnosis and classification (Table 2).

The objective of this review is to focus on anomalies originating from the coronary arteries of the aorta but from a different Valsalva sinus (from opposite sinuses), which have challenged pediatric and adult cardiologists.

Table 1 - Coronary artery normal pattern in humans.

\begin{tabular}{|c|c|}
\hline Characteristic & Variation \\
\hline \multicolumn{2}{|l|}{ Number of ostia } \\
\hline Location & Right and left (anterior) \\
\hline Proximal orientation & $45^{\circ}$ at $90^{\circ}$ in the aortic wall \\
\hline Proximal trunk & $\begin{array}{l}\text { Left only (anterior descending and circumflex } \\
\text { arteries) }\end{array}$ \\
\hline Proximal course & Straight from the ostium to the end \\
\hline Mean course & Extramural (subepicardial) \\
\hline Branches & Suitable for dependent myocardium \\
\hline Essential territories & $\begin{array}{l}\text { Right coronary (free wall of the right } \\
\text { ventricle), anterior descending artery (antero- } \\
\text { septal wall), marginal obtuse (free wall of the } \\
\text { left ventricle) }\end{array}$ \\
\hline End & Capillary bed \\
\hline
\end{tabular}


Table 2 - Classification of congenital coronary artery anomalies.

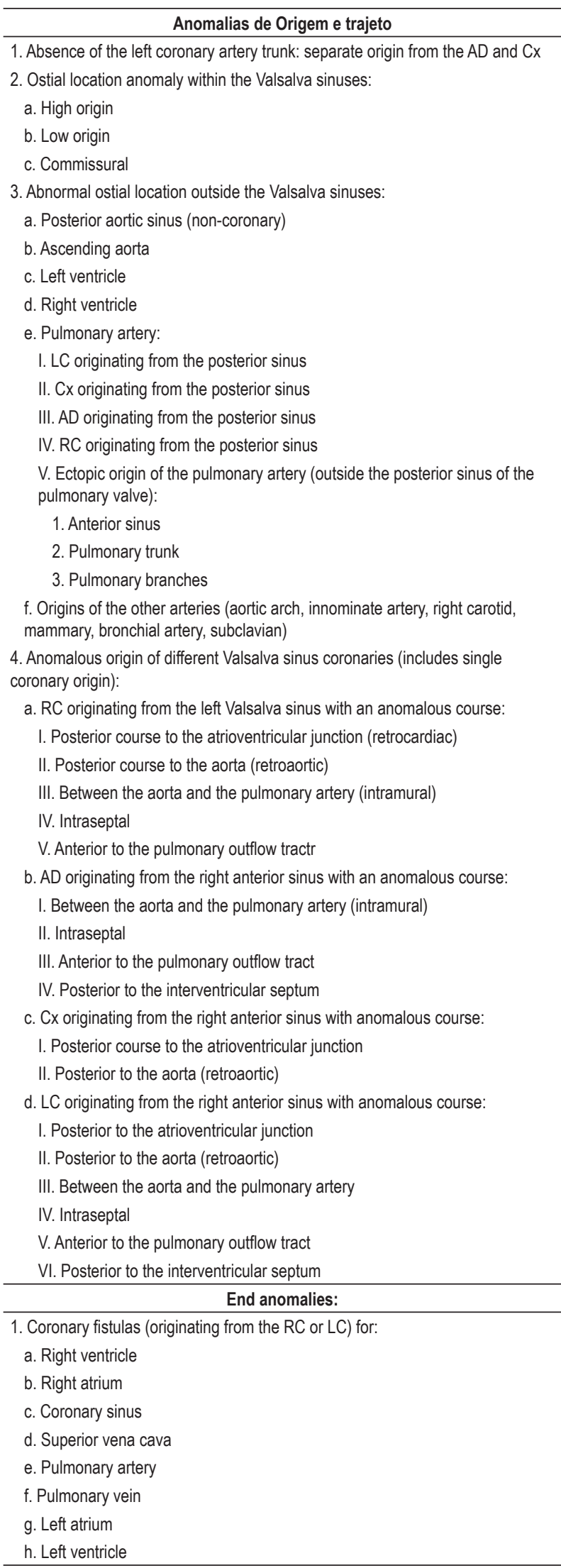

\section{How should equipment be adjusted to assess coronary anatomy?}

Advanced equipment has favored the diagnosis of structures such as the coronary arteries. Some equipment comes with specific settings for this assessment that adjust automatically. However, some tips or basic rules can be very useful and improve diagnostic accuracy as follows:

Use tranducers with high-frequency as often as possible, even in adult patients (pediatric transducers with a frequency $>8 \mathrm{MHZ}$ ); focus on the coronary arteries in the field near the sector.

Decrease compression (to enable more distinct identification of the coronary arteries).

Use the long axis/short axis parasternal window possibly one intercostal space upward: rotate it clockwise toward the left coronary artery and counterclockwise toward the right coronary artery, pointing toward the right shoulder. All movements must be very subtle.

Never use still imaging for diagnostic purposes: Due to the size of the arteries and the possibility of false diagnosis, it is important to evaluate them very slowly, usually frame by frame. A real-time assessment is not ideal as an important translation of the coronary arteries occurs during the cardiac cycle.

Color Doppler evaluation is mandatory (with speed adjustments).

\section{What to assess for anomalies originating from the coronary arteries of the opposite sinuses?}

Anomalies originating from opposite sinuses occur in around $1 \%$ of the general population. ${ }^{1}$ One of the great challenges is that most patients remain asymptomatic; when they become symptomatic, most present with a more serious event. Right coronary anomalies originating from the left sinus are more frequent; however, left coronary anomalies originating from the right sinus have the greatest risk for ischemic events. Anatomical risk factors include: An interarterial and intramural course, slit ostium, and acute emergence angle tangent to the vessel wall. All of these possibilities increase the risk for ischemic events, ventricular arrhythmias, and sudden death. Exhausting physical exercise increases the chance of sudden death, making this entity the second cause of sudden death in young athletes after hypertrophic cardiomyopathy. ${ }^{2}$

Despite controversies and discussions about athlete screening protocols, the echocardiographer should be prepared to make this diagnosis as it is the method of choice for this population. TTE has excellent diagnostic accuracy at detecting the ostium and the proximal course of the coronary arteries. ${ }^{6}$ However, as it is operator-dependent, the expertise of this professional makes this cardiovascular imaging modality the key piece in an algorithm for diagnosing this congenital heart disease.

To assess diagnostic quality and establish guidelines and algorithms, a multicenter study reviewed the vulnerable points of echocardiographers in recognizing the most important anatomical markers. ${ }^{3,7}$ 
Interarterial course of the coronary arteries

When emerging from the left sinus, the initial epicardial course of the normal left coronary artery is short (in adults $<4 \mathrm{~cm}$ ) before dividing into circumflex (Cx) and anterior descending (AD) arteries. The RC originates from the right Valsalva sinus and runs behind the pulmonary artery, below the right atrial appendage, and along the right atrioventricular groove (Figure 1).

There are several alternative courses anomalous according to the origin of the coronary arteries (Figure 2). The anomaly originating from the LC of the right sinus and the RC originating from the left sinus allow an interarterial course (between the aorta and the pulmonary artery). In both, the coronary artery may originate from a single ostium or from separate ostia. (Figure 3).

The echocardiographic image in the short-axis parasternal plane, with the transducer pointed at the patient's left shoulder, delimits the initial course between the two vessels, with a space (echogenic) separating it from the arterial wall (Figure 4). Another very important detail is the ostium position in relation to the commissure. The ideal position would be equidistant (in the middle of the Valsalva sinus), as it would reduce the risk of ostial stenosis and, in cases of surgical treatment, would facilitate the surgical technique. It is important to highlight that the visualization of the coronary commissures and ostia with very subtle movement indirectly informs that there is no high coronary origin (above the sinotubular junction [ST]]).

\section{Intramural course}

The second anatomical marker, and perhaps the most important as a predictor of ischemic events, is the proximal $\mathrm{RC}$ or LC intramural course originating from the opposite sinus. Unfortunately, it is also the most diagnostically challenging. Histologically, it is defined as sharing of the tunica media by the coronary artery and the aorta (Figure 5). An ECHO diagnosis is made using the same twodimensional image associated with color Doppler, which is mandatory (Figures 6 and 7). The first characteristic is the angulation of the proximal portion of the coronary artery at an acute angle ( $<30$ degrees) and the diastolic flow in the proximal course located "inside" the wall of the aorta (Video 1). One of the most remarkable and specific characteristics is called diastolic "hang-up" (Videos 2 and 3), which means the final diastolic flow in this portion, practically when it would no longer have anterograde flow. In addition to being essential for diagnosis, color Doppler should be explored and, often, subtle changes in the direction of the ultrasound beam can enhance the flow tangent to the aortic wall. Always remember to adjust the speed of the color flow mapping. A pulsed Doppler pattern is also valuable despite technical, angulation, and translation of the heart difficulties. As for anatomical markers, the presence of separated ostia is related to a higher incidence of an intramural course. ${ }^{8}$ An origin very close to the commissure must also be reported, as it would be a non-compliant area contributing to flow restriction.

\section{High origin of the coronary arteries}

The coronary ostia follow a normal pattern, which is usually equidistant from the commissures below the STJ. A high origin is identified when the origin is above the STJ (Figure 8). This anomaly alone would not represent risk of ischemic events; however, the possibility of an ostial anomaly or compression, or even of an acute angle at the origin ( $<45$ degrees) can be associated with high output. Echocardiographically, the standard long-axis parasternal plane requires some modification, with an angle of around 30 degrees toward the patient's right shoulder (Video 4).

\section{What is the role of multimodality cardiovascular imaging in congenital coronary artery anomalies?}

In the current era, multimodality is already well established and used precisely to complement and

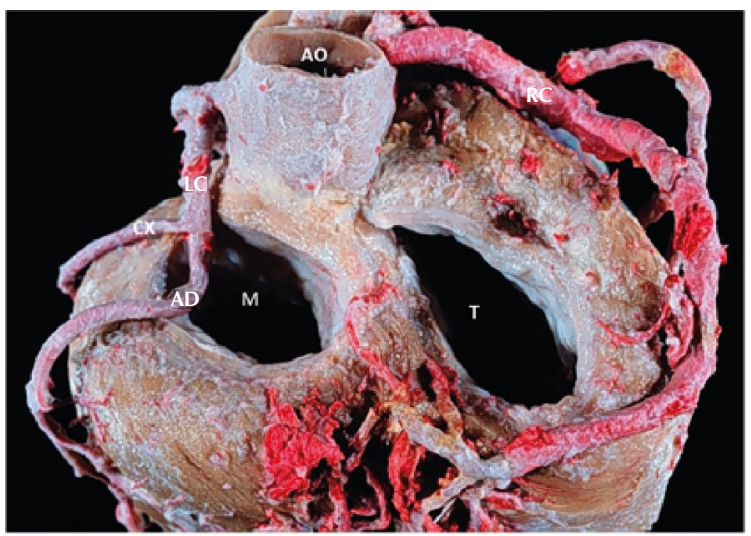

$A D$ : anterior descending artery; AO: aorta; Cx: circumflex artery; LC: left coronary artery; M: mitral valve; RC: right coronary artery; T: tricuspid valve

Figure 1 - Normal coronary artery anatomy showing the main branches and epicardial distribution. 


\section{Editorial}
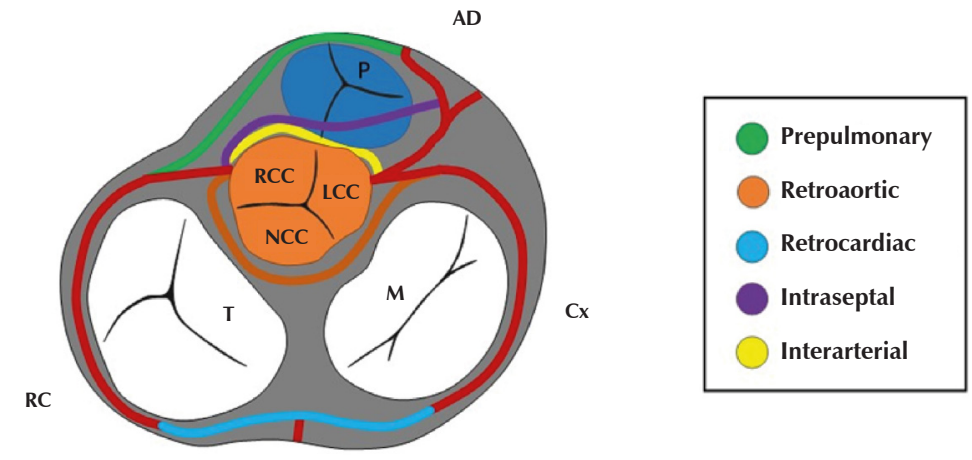

$A D$ : anterior descending artery; CX: circumflex artery; LC: left coronary artery; LCC: left coronary cusp; M: mitral valve.RC: right coronary artery; RCC: right coronary cusp; NCC: non-coronary cusp; T: tricuspid valve.

Figure 2 - Possible coronary artery courses.

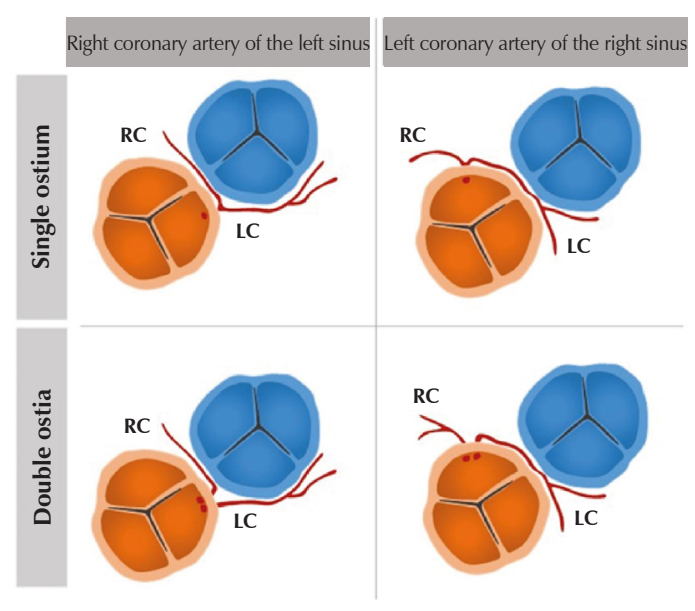

LC: Left coronary artery; RC: Right coronary artery .

Figure 3 - Main anomalies originating from the opposite sinuses.

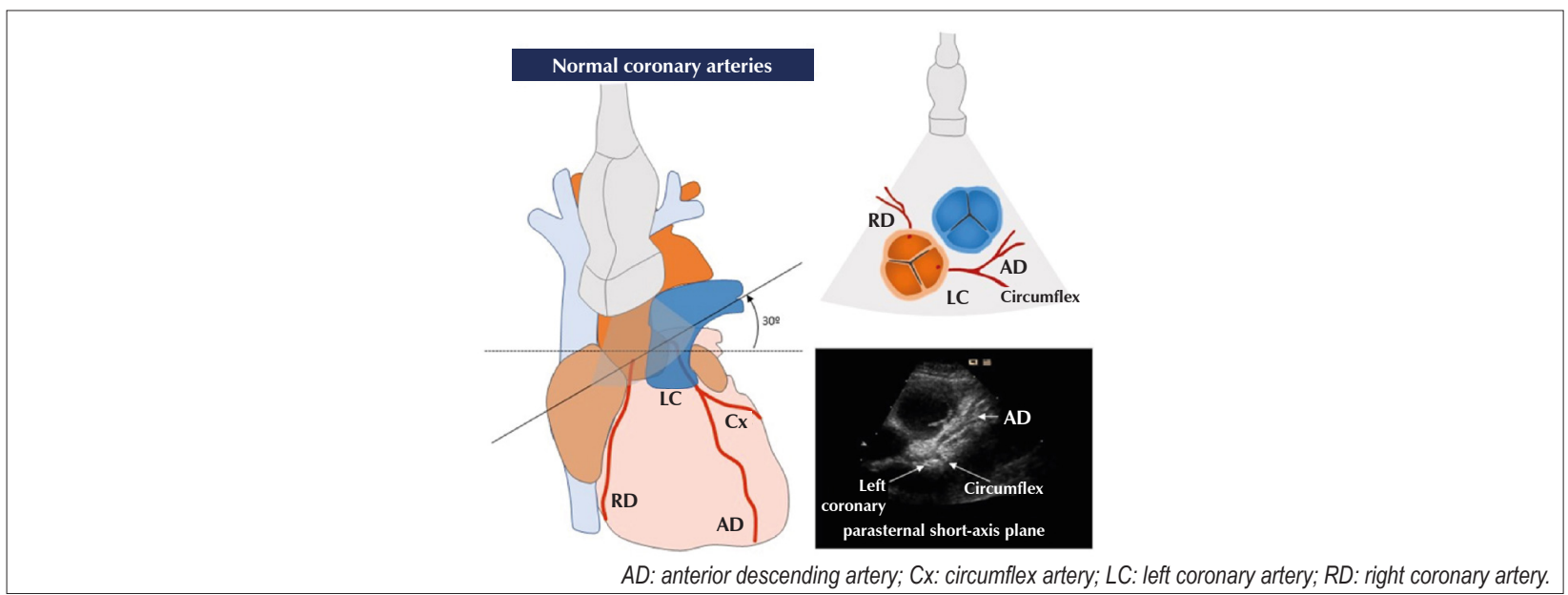

Figure 4 - Orientation of the ultrasound beam plane used to obtain the echocardiographic image of the coronary arteries. Short-axis echocardiographic image showing the initial course of the left coronary artery and the bifurcation. 


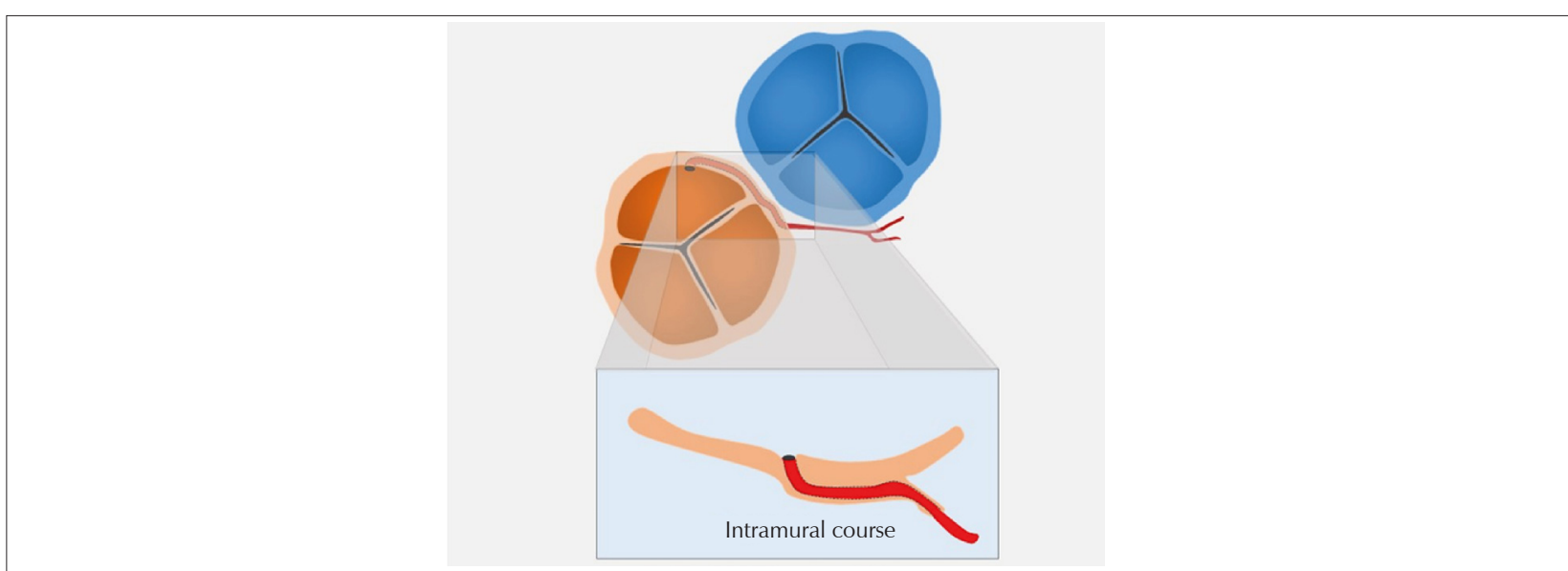

Figure 5 - Representation of the interarterial and intramural courses of the left coronary artery originating from the right sinus.
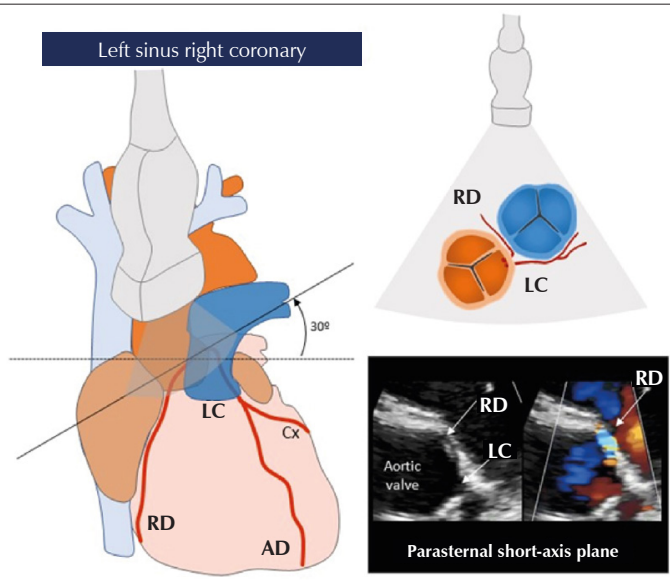

$A D$ : anterior descending artery; $C x$ : circumflex artery; LC: left coronary artery; RC: right coronary.

Figure 6 - Orientation of the ultrasound beam plane used to obtain the echocardiographic image of the coronary arteries. Short-axis echocardiographic image showing the initial course of the right coronary artery originating from the left sinus in the "Color compare" mode. The color Doppler image showed the right coronary artery flow practically within the aortic wall: interarterial and intramural course.

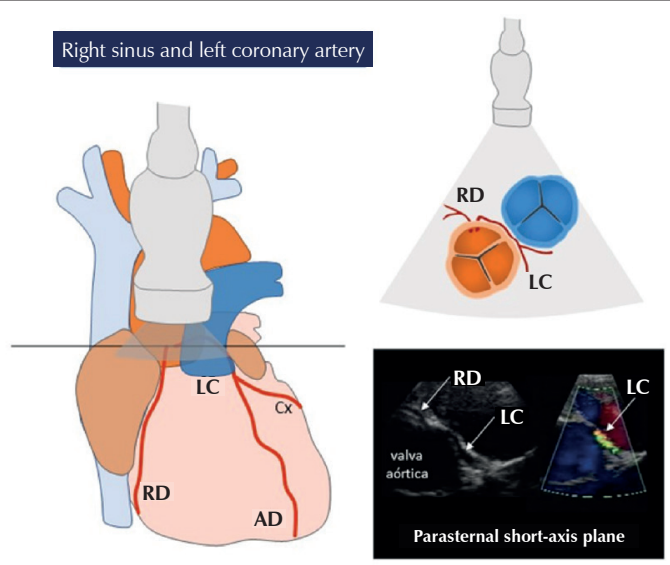

$A D$ : anterior descending artery; Cx: circumflex artery; LC: left coronary artery; RD: right coronary artery.

Figure 7 - Orientation of the ultrasound beam plane used to obtain the echocardiographic image of the coronary arteries. Short-axis echocardiographic image showing the initial course of the left coronary artery originating from the left sinus in the "Color compare" mode. 


\section{Editorial}

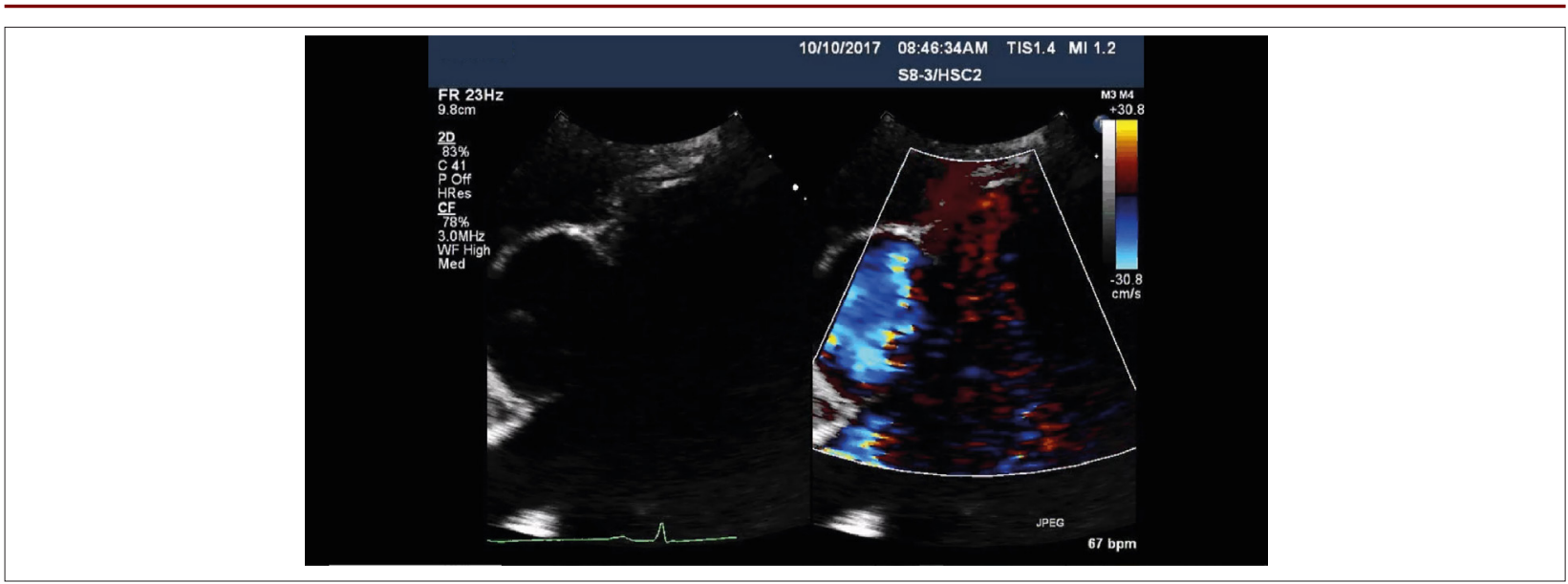

Video 1 - Parasternal short axis view, in Color Doppler compare mode: Anomalous origin of the left coronary artery from the right sinus with interarterial and intramural course. There is an angle < 30 degrees of the coronary artery in relationship with aortic wall. There is a late color demonstration of the intramural and interarterial course of the coronary artery ("hang-up").

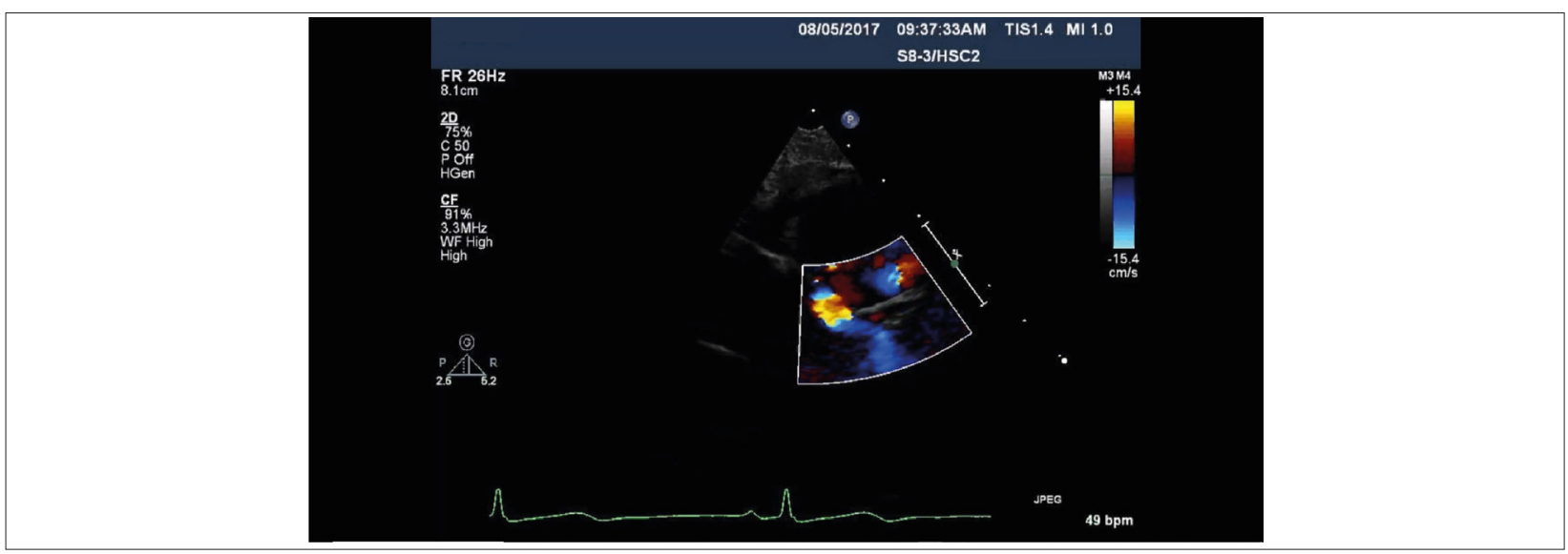

Video 2 - Parasternal short axis view with Color Doppler: Anomalous origin of the left coronary artery from the right sinus with interarterial and intramural course. There is an angle < 30 degrees of the coronary artery in relationship with aortic wall, however, there is a shorter course than the case 1. There is a late color demonstration of the intramural and interarterial course of the coronary artery ("hang-up").

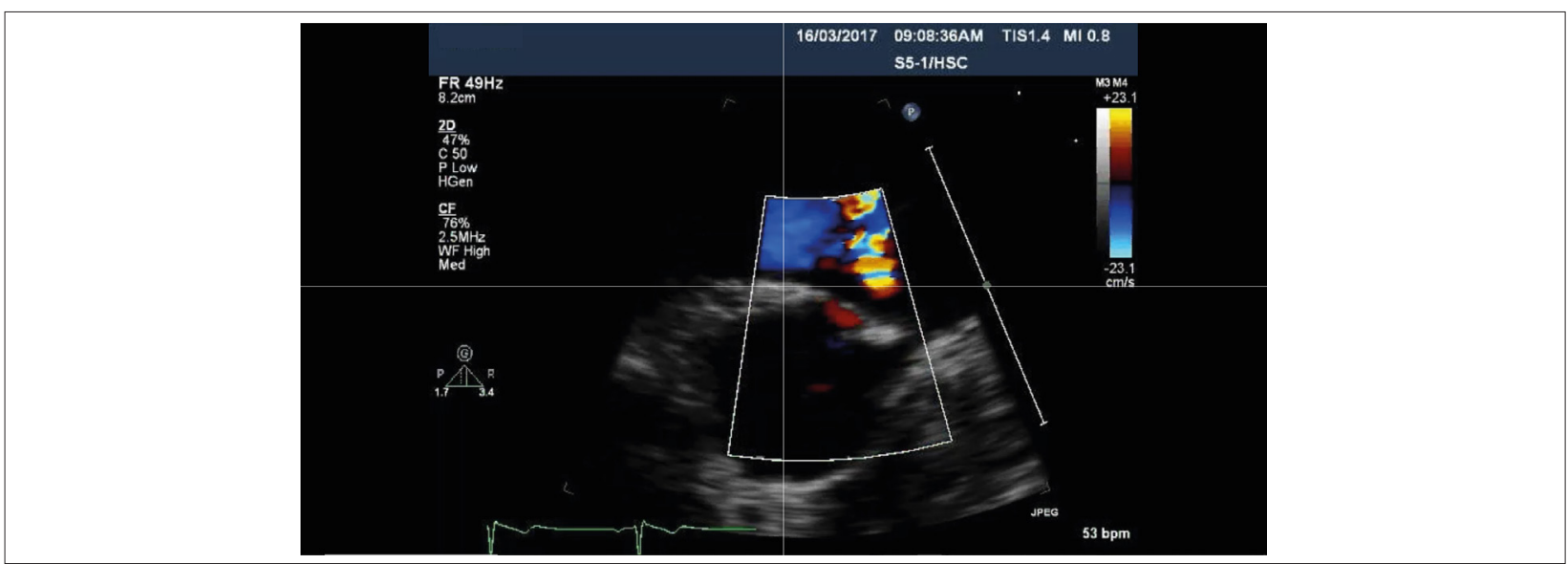

Video 3 - Parasternal short axis view with Color Doppler: Anomalous origin of the right coronary artery from the left sinus with interarterial and intramural course. There is an angle < 30 degrees of the coronary artery in relationship with aortic wall. There is a late color demonstration of the intramural and interarterial course of the coronary artery ("hang-up"). Also, the ostio of the left coronary artery located very close, both close to the comissure between the right and left cusp of the aortic valve. 
help establish an assessment and definition algorithm for possible therapeutic decision-making that may even be surgical. ${ }^{3}$

The role of the pediatric cardiologist and echocardiographer is consolidated within the circle of care even for adult patients, especially young athletes. National and international guidelines are unanimous in establishing the role of these professionals as a pillar for an appropriate diagnosis of these anomalies and adequate conduct, especially for young athletes whose future depends on cardiovascular system integrity. ${ }^{9}$

\section{Acknowledgments}

The authors thank Dr. Carlos Vilalobos, fellow in Pediatric Cardiology at Sickkids, Toronto, for creating the diagrams and figures included in the text.

They also thank Dr. Vera Aiello, head of the Instituto do Coração Laboratory of Pathology at FMUSP, for her contribution of the anatomy images.

\section{Conflict of interest}

The author declares no conflicts of interest.

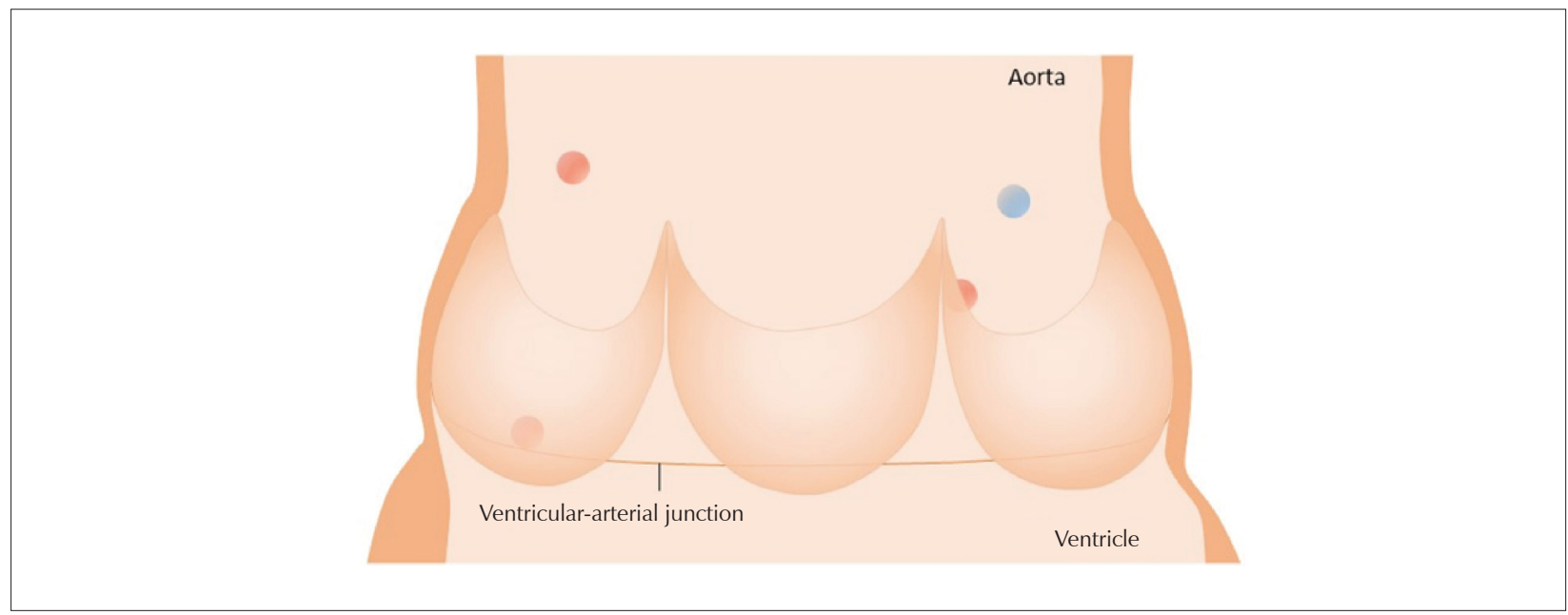

Figure 8 - Possible ectopic locations of the coronary artery ostia (in red) versus the usual location (in blue).

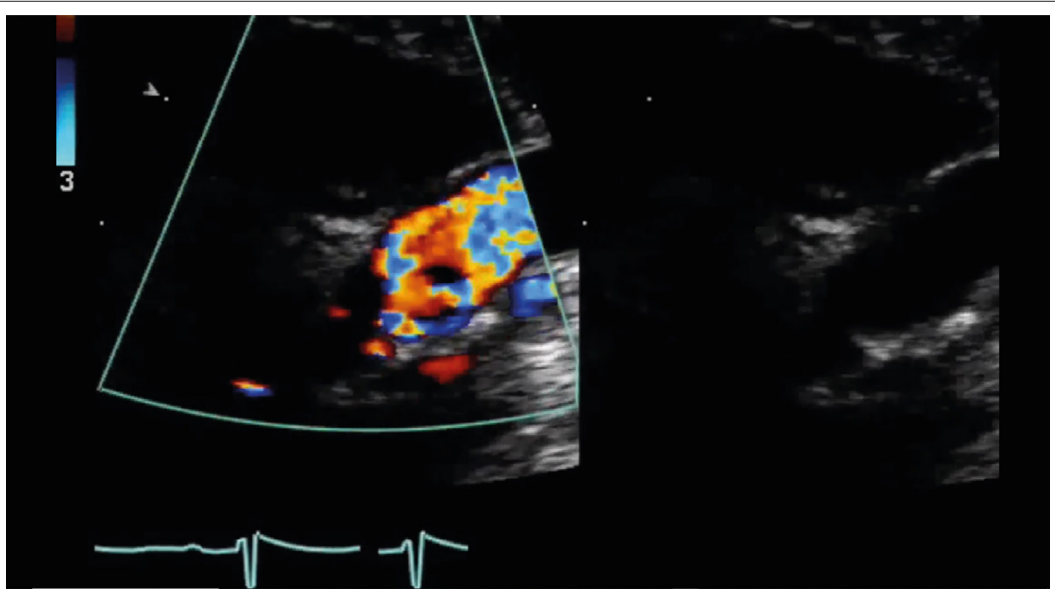

Video 4 - Parasternal short axis view with Color Doppler: High-take off of the right coronary artery above of the sinotubular junction.

\section{References}

1. Basso C, Maron BJ, Corrado D, Thiene G. Clinical profile of congenital coronary artery anomalies with origin from the wrong aortic sinus leading to sudden death in young competitive athletes. J Am Coll Cardiol. 2000;35(6):1493-501. doi: http://dx.doi.org/10.1016/S0735-1097(00)00566-0
2. Maron BJ, Haas TS, Ahluwalia A, Murphy CJ, Garberich RF. Demographics and epidemiology of sudden deaths in young competitive athletes: From the United States National Registry. Am J Med. 2016;129(11):1170-7. doi: http://dx.doi.org/10.1016/j.amjmed.2016.02.031 


\section{Editorial}

3. Gräni C, Buechel RR, Kaufmann PA, Kwong RY. Multimodality imaging in individuals with anomalous coronary arteries. JACC Cardiovasc Imaging. 2017;10(4):471-81. doi: http://dx.doi.org/10.1016/j.jcmg.2017.02.004

4. Stout KK, Daniels CJ, Aboulhosn JA, Bozkurt B, Broberg CS, Colman JM, etal. 2018 AHA/ACC Guideline for the Management of Adults With Congenital Heart Disease: A Report of the American College of Cardiology/American Heart Association Task Force on Clinical Practice Guidelines. Circulation. 2019;139:698-800. doi: https://doi.org/10.1161/CIR.0000000000000603

5. Angelini P. Coronary artery anomalies: An entity in search of an identity. Circulation. 2007;115(10):1296-305.

6. Weiner RB, Wang F, Hutter AM, Wood MJ, Berkstresser B, McClanahan C, et al. The feasibility, diagnostic yield, and learning curve of portable echocardiography for out-of-hospital cardiovascular disease screening. J Am Soc Echocardiogr. 2012;25(5):568-75. doi: http://dx.doi.org/10.1016/j. echo.2012.01.010
7. Lorber R, Srivastava S, Wilder TJ, McIntyre S, Decampli WM, Williams WG, et al. Anomalous aortic origin of coronary arteries in the young echocardiographic evaluation with surgical correlation. JACC Cardiovasc Imaging. 2015;8(11):1239-49. doi: 10.1016/j.jcmg.2015.04.027. Erratum in: JACC Cardiovasc Imaging. 2016;9(2):217.

8. Mainwaring RD, Reddy VM, Reinhartz O, Petrossian E, Punn R, Hanley FL. Surgical repair of anomalous aortic origin of a coronary artery. Eur J Cardiothoracic Surg. 2014;46(1):20-6. doi: 10.1093/ejcts/ezt614

9. Baggish AL, Battle RW, Beaver TA, Border WL, Douglas PS, Kramer CM, Martinez MW, Mercandetti JH, Phelan D, Singh TK, Weiner RB, Williamson E. Recommendations on the use of multimodality cardiovascular imaging in young adult competitive athletes: a report from the american society of echocardiography in collaboration with the society of cardiovascular computed tomography and the society for cardiovascular magnetic resonance. J Am Soc Echocardiogr. 2020;33(5):523-49. doi: 10.1016/j. echo.2020.02.009 